\title{
Long-term ethanol intoxication reduces inflammatory responses in rats
}

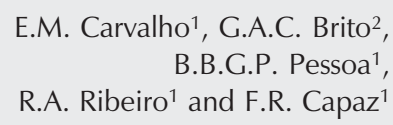

\author{
Departamentos de ${ }^{1}$ Fisiologia e Farmacologia, and ${ }^{2}$ Morfologia, \\ Universidade Federal do Ceará, Fortaleza, CE, Brasil
}


Nevertheless, the mechanism and the role of other immune-inflammatory cells (i.e., macrophages, lymphocytes, eosinophils, mast cells) in this process are not completely understood.

Ethanol inhibits the production of inflammatory cytokines through alveolar macrophages in chronically ethanol-fed mice $(5,9,10)$ and suppresses lipopolysaccharideinduced expression of inducible nitric oxide synthase $(11,12)$, and tumor necrosis factor $\alpha(\mathrm{TNF}-\alpha)$ production. Recently, it was also reported that ethanol inhibits mast cell degranulation and TNF- $\alpha$ production (13).

Most studies addressing alcohol-induced immune suppression have focused on either acute or chronic alcohol treatment. However, few studies have shown immune impairment during the withdrawal period that follows interruption of long-term exposure. Therefore, we investigated the inflammatory response in rats during ethanol treatment and withdrawal using the inhalation route, which is known to be an efficient method to obtain a constant and high serum ethanol concentration (14) and which has been extensively used in recent ethanol studies with rodents (15-17). We also investigated the modulation of mast cell degranulation in this animal model.

\section{Material and Methods}

\section{Animals}

Male Wistar rats (180-200 $\mathrm{g}$ body weight) were housed in temperature-controlled rooms and received water and food ad libitum. The ethical guidelines described in the National Institutes of Health Guide for Care and Use of Laboratory Animals were followed throughout the experiments.

\section{Drugs}

The following drugs were used: carrageenan (Biochemical Products, Indianapo- lis, IN, USA), dextran 70 (Pharmacia/Pfizer, Belo Horizonte, MG, Brazil), toluidine blue, compound 48/80, and Sephadex (Sigma, St. Louis, MO, USA). All other chemicals were of analytical grade or equivalent.

\section{Ethanol administration}

The rats were kept in acrylic boxes (52 $\mathrm{x}$ $34 \times 27 \mathrm{~cm})$, where ethanol $(99.5 \%$, v/v) was delivered by an infusion pump at a constant rate of $97 \mathrm{mg} / \mathrm{min}$ onto a filter paper wick in a flask. A respirator pump delivered air through the flask into the chamber at 3.5 to 7 liters/min. The airflow was controlled with a calibrated flow meter. The rats were exposed to an increasing concentration of ethanol vapor over a 10-day period (14).

After the 10-day exposure period the rats were removed from the chamber and observed for signs of withdrawal. Ethanol withdrawal was assessed using the single sign called "convulsion on handling". Rats were scored hourly for convulsions during a 14-h period according to a system modified from Goldstein (18). A rat was lifted by the tail and observed for convulsions, and if no convulsions were observed the animal was gently spun $180^{\circ}$ and examined. A scale of $0-2$ was used as follows: 2, convulsions when the animal was lifted by the tail, often with onset delayed by as much as 1-2 s; 1 , no convulsions when the animal was lifted by the tail, but convulsions after a gentle spin through $180^{\circ} ; 0.5$, only a facial grimace after spinning, and 0 , no convulsions. More than $90 \%$ of the rats exposed to ethanol vapor presented tremors, limb extension or convulsions within $7 \mathrm{~h}$ after withdrawal (mean score $\pm \mathrm{SEM}=1.72 \pm 0.32$ ), confirming that the dose was adequate for producing dependence on ethanol (14). One group of animals was evaluated immediately upon removal from the chambers (treated group intoxicated), and another group was studied $7 \mathrm{~h}$ after the interruption of ethanol inhalation (withdrawn group - withdrawn). 


\section{Air pouches}

A subcutaneous 6-day-old air pouch was produced as described by Edwards et al. (19). Briefly, on the 4th day of ethanol inhalation, the dorsal region of the rats was shaved and $20 \mathrm{ml}$ of sterile air was injected subcutaneously. Three days later, $10 \mathrm{ml}$ of sterile air was again injected into the air pouch to maintain the patency of the cavity. On the 10 th day of ethanol inhalation (6 days after the initial injection of air), $500 \mu \mathrm{g} / \mathrm{ml}$ carrageenan was injected into the pouch $6 \mathrm{~h}$ before removal from the chamber (treated group). In the withdrawal group, $500 \mu \mathrm{g} / \mathrm{ml}$ carrageenan was injected into the pouch $1 \mathrm{~h}$ after removal from the chamber. The animals were sacrificed $6 \mathrm{~h}$ after the carrageenan injection. Control animals did not inhale ethanol and had saline or carrageenan injected into the air pouch. The pouch was washed with $5 \mathrm{ml}$ phosphate-buffered saline (PBS) containing heparin $(5 \mathrm{IU} / \mathrm{ml})$ and $3 \%$ bovine serum albumin (BSA). The washout fluid was collected and total and differential leukocyte counts were performed by the method of Ribeiro et al. (20).

\section{Peritoneal cavity}

The treated and withdrawal groups were treated with Sephadex (1.5 mg, $1 \mathrm{ml}$, ip). Control rats were treated with saline $(1 \mathrm{ml}, \mathrm{ip})$ or Sephadex (1.5 mg, ip), but ethanol vapor was not administered. The animals were sacrificed $24 \mathrm{~h}$ after Sephadex administration, and the peritoneal cavity was washed with $10 \mathrm{ml}$ PBS containing heparin $(5 \mathrm{IU} / \mathrm{ml})$ and $3 \%$ BSA. Total and differential leukocyte counts in the lavage fluid were performed as described by Ribeiro et al. (20).

\section{Hind paw edema}

Paw edema was induced by subplantar injection of carrageenan (300 $\mu \mathrm{g} / \mathrm{paw})$ or dextran $(100 \mu \mathrm{g} / \mathrm{paw})$ in a final volume of
$0.1 \mathrm{ml}$ into the right hind paw of rats. All drugs were dissolved in sterile saline. Control animals received sterile saline $(0.1 \mathrm{ml} /$ paw). Paw edema was measured with a hydroplethysmometer (Ugo Basile 7140, Plethysmometer, Varese, Italy) immediately before and 1, 2, 3, and $4 \mathrm{~h}$ after the carrageenan or dextran challenges. The increase in paw volume (edema volume) was obtained by subtracting the paw volume measured before stimulus injection (21). The area under the time-course curve was calculated using the trapezoidal rule, and results are reported in arbitrary units.

\section{Leukogram}

With each animal serving as its own control, a leukogram was obtained before (day 0 ) and on the 5th, 9th and 10th day of ethanol inhalation. Total and differential white blood cell counts were performed on blood samples collected from a tail vein using a Neubauer chamber and blood smears stained with a HEMA $3^{\text {TM }}$ Stain Set (Pittsburgh, PA, USA).

\section{Mast cell depletion}

After the ethanol treatment, the animals were killed by cervical dislocation, a laparotomy was performed and segments of mesentery were removed and incubated with 20 ml Locke-Ringer's solution, or Locke-Ringer's solution plus compound 48/80 $(0.8 \mu \mathrm{g} / \mathrm{ml})$ for $30 \mathrm{~min}$ at room temperature. Compound 48/80 $(0.8 \mu \mathrm{g} / \mathrm{ml})$ was used to induce mast cell degranulation. After the incubation period, the tissues were placed on microscope slides, stained with toluidine blue for $2 \mathrm{~min}$ and then washed with distilled water and allowed to dry. The percent of mast cell degranulation was determined by counting 100 cells using a conventional light microscope.

\section{Statistical analysis}

Data are reported as means \pm SEM. Sta- 
Figure 1. Effect of ethanol inhalation on paw edema induced by carrageenan. Rat paw edema was induced by intraplantar injection of carrageenan $(\mathrm{Cg}, 300$ $\mu \mathrm{g} / 0.1 \mathrm{ml}$ ) in animals exposed to ethanol vapor for 10 days (intoxicated or treated group, Trt) and animals pretreated with ethanol and submitted to $7 \mathrm{~h}$ of withdrawal (withdrawn group, Wth). A, Edema was measured at 1, 2 3 , and $4 \mathrm{~h}$ after the inflammatory challenge and is reported as the mean \pm SEM of the increase in paw volume (ml) compared to basal volume for 5-6 animals per group. $B$, The area under the curve was determined using the trapezoidal rule. Data are reported as mean \pm SEM for 5-6 animals per group. Control animals (-) received $\mathrm{Cg}$ but no ethanol vapor. The saline group (Sal) received only saline $(0.1 \mathrm{ml})$. ${ }^{*} P$ $<0.05$ compared to control (-): $+P<0.05$ compared to the saline group (ANOVA followed by the Bonferroni test).

Figure 2. Effect of ethanol inhalation on paw edema induced by dextran. Rat paw edema was induced by intraplantar injection of dextran (Dx, $300 \mu \mathrm{g} / 0.1 \mathrm{ml}$ ) in animals exposed to ethanol vapor for a 10-day period (intoxicated or treated group, Trt) and animals pretreated with ethanol and submitted to $7 \mathrm{~h}$ of withdrawal (withdrawn group, Wth). A, Edema was measured at 1, 2, 3 , and $4 \mathrm{~h}$ after the inflammatory challenge and is reported as the mean \pm SEM of the increase in paw volume (ml) compared to basal volume for 5-6 animals per group. $B$, The area under the curve was determined using the trapezoidal rule. Data are reported as means \pm SEM for 5-6 animals per group. Control animals (-) received Dx, but no ethanol vapor. The saline group (Sal) received only saline $(0.1$ $\mathrm{ml}) .{ }^{*} \mathrm{P}<0.05$ compared to control $(-) ;+P<0.05$ compared to the saline group (ANOVA followed by the Bonferroni test).
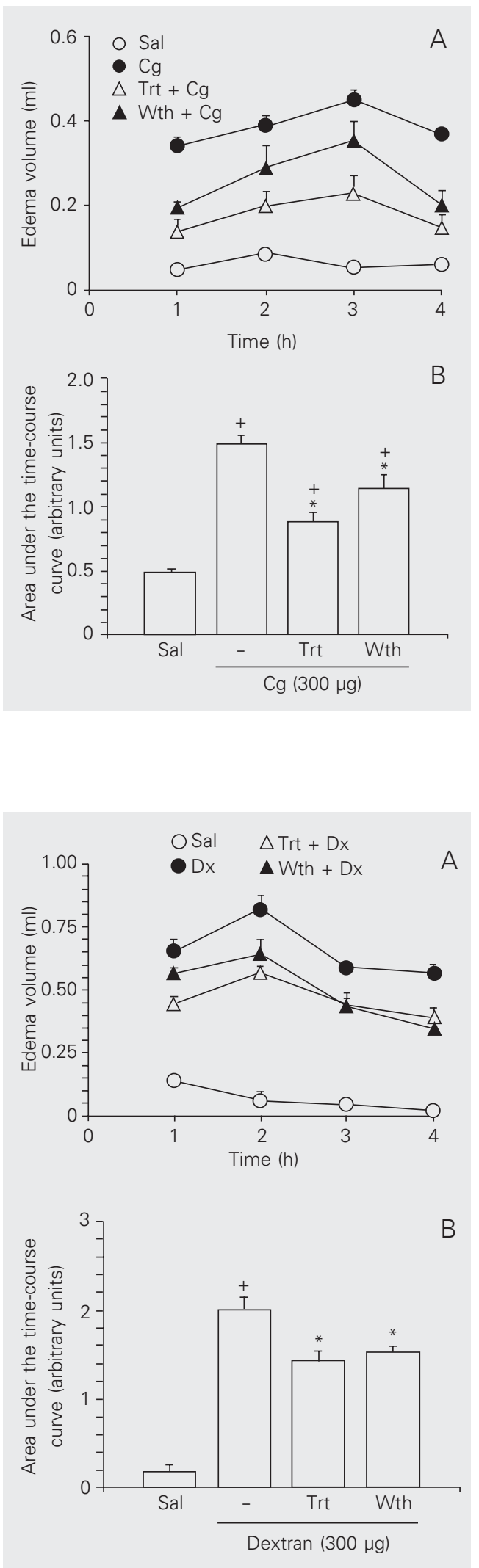

tistical evaluation was performed by analysis of variance (ANOVA) followed by the Bonferroni test. Statistical differences were considered significant at $\mathrm{P}<0.05$.

\section{Results}

\section{Paw edema}

Subplantar injection of carrageenan induced a progressive paw edema that reached a maximal value by $3 \mathrm{~h}$. The edema induced by carrageenan was significantly reduced by long-term ethanol inhalation in both the intoxicated and withdrawn groups (62 and $35 \%$ inhibition, respectively, $\mathrm{P}<0.05$; Figure 1). Dextran induced a more intense paw edema of more rapid onset which peaked at $2 \mathrm{~h}$ and remained elevated up to $4 \mathrm{~h}$ after injection. The edema induced by dextran was also significantly reduced by long-term ethanol inhalation in both groups tested (intoxicated: $32.2 \%$ inhibition; withdrawn: $26.4 \%$ inhibition, $\mathrm{P}<0.05$; Figure 2 ).

\section{Neutrophil and eosinophil migration}

Long-term ethanol inhalation effectively reduced carrageenan-induced neutrophil migration into a subcutaneous 6-day-old air pouch (intoxicated group: 95\%; withdrawn group: $41 \%, \mathrm{P}<0.05)$ compared to control animals (Figure 3A). Similar significant inhibition was observed in Sephadex-induced eosinophil migration into the peritoneal cavity (intoxicated group: 100\%; withdrawn group: $64 \%, \mathrm{P}<0.05$; Figure $3 \mathrm{~B}$ ).

\section{Mast cell degranulation}

Compound 48/80 is known to induce extensive mast cell degranulation (22). Longterm ethanol inhalation produced a significant decrease in the percentage of compound 48/80-induced mast cell degranulation (positive control: $82.2 \% v s$ intoxicated: $49.1 \%$ and withdrawn: $51.1 \%$ degranulated mast 
cells, $\mathrm{P}<0.05$; Figure $3 \mathrm{C}$ ).

\section{Peripheral blood leukocytes}

The leukogram performed during ethanol treatment showed a progressive increase in total leukocyte and neutrophil counts throughout the 10 days of observation. Both cell types were already significantly increased from the 5th day and reached a peak on the 10th day $(\mathrm{P}<0.05$; Figure 4$)$. Even $7 \mathrm{~h}$ after interruption of ethanol administration, the leukocyte and neutrophil counts, although

Figure 3. Effect of ethanol inhalation on carrageenaninduced neutrophil migration into a 6-day-old subcutaneous air pouch $(A)$, on Sephadex-induced eosinophil migration into the rat peritoneal cavity (B) and on compound 48/80-induced mast cell degranulation in the rat mesentery $(C)$. Animals were exposed to ethanol vapor for 10 days (intoxicated or treated group, Trt) and a second group was exposed to ethanol vapor for 10 days followed by $7 \mathrm{~h}$ of withdrawal (withdrawal group, Wth). Panel A, Carrageenan ( $\mathrm{Cg}, 500 \mu \mathrm{g}$ ) was injected into the air pouch of animals of the intoxicated and withdrawal groups and neutrophil migration was determined $6 \mathrm{~h}$ after the carrageenan challenge. Control animals (-) received carrageenan but no ethanol vapor. The saline group (Sal) received only saline $(1.0 \mathrm{ml} /$ cavity). Data are reported as the mean \pm SEM of the number of neutrophils $\times 10^{6} / \mathrm{ml}$ for $5-6$ rats per group. ${ }^{*} P<0.05$ compared to control $(-)$; $+P<0.05$ compared to the saline group (Sal; ANOVA followed by the Bonferroni test). Panel $B$, Eosinophil migration was determined $24 \mathrm{~h}$ after the Sephadex challenge. Control animals (-) received Sephadex but no ethanol vapor. The saline group (Sal) received only saline $(1.0 \mathrm{ml} /$ cavity). Data are reported as the mean \pm SEM of the number of eosinophils $\times 106 / \mathrm{ml}$ for 6 rats per group. ${ }^{*} P<0.05$ compared to control $(-) ;+P<0.05$ compared to the saline group (ANOVA followed by the Bonferroni test). Panel $C$, The mesenteries of rats exposed to ethanol vapor were incubated with compound $48 / 80(0.8 \mu \mathrm{m} /$ $\mathrm{ml}$ ) in Locke-Ringer's solution for $30 \mathrm{~min}$. In the positive control group (-), the animals were not submitted to ethanol vapor. The mesentery of control rats (LRs) not submitted to ethanol vapor was incubated with Locke-Ringer's solution without compound 48/80. Data are reported as the mean \pm SEM of the percentage of mast cells undergoing degranulation, for 6-8 animals per group. ${ }^{*} P<0.05$ compared to the positive control group (-); $+P<0.05$ compared to the control group (LRs) (ANOVA followed by the Bonferroni test). decreasing, were still significantly increased when compared to initial values.

\section{Discussion}

Acute and chronic ethanol administration has long been known to produce a variety of pathologic tissue changes. In order to study and understand the mechanisms underlying ethanol addiction and withdrawal, efforts have been made to reproduce some of these pathologic changes in experimental models. Several in vivo studies have demon-

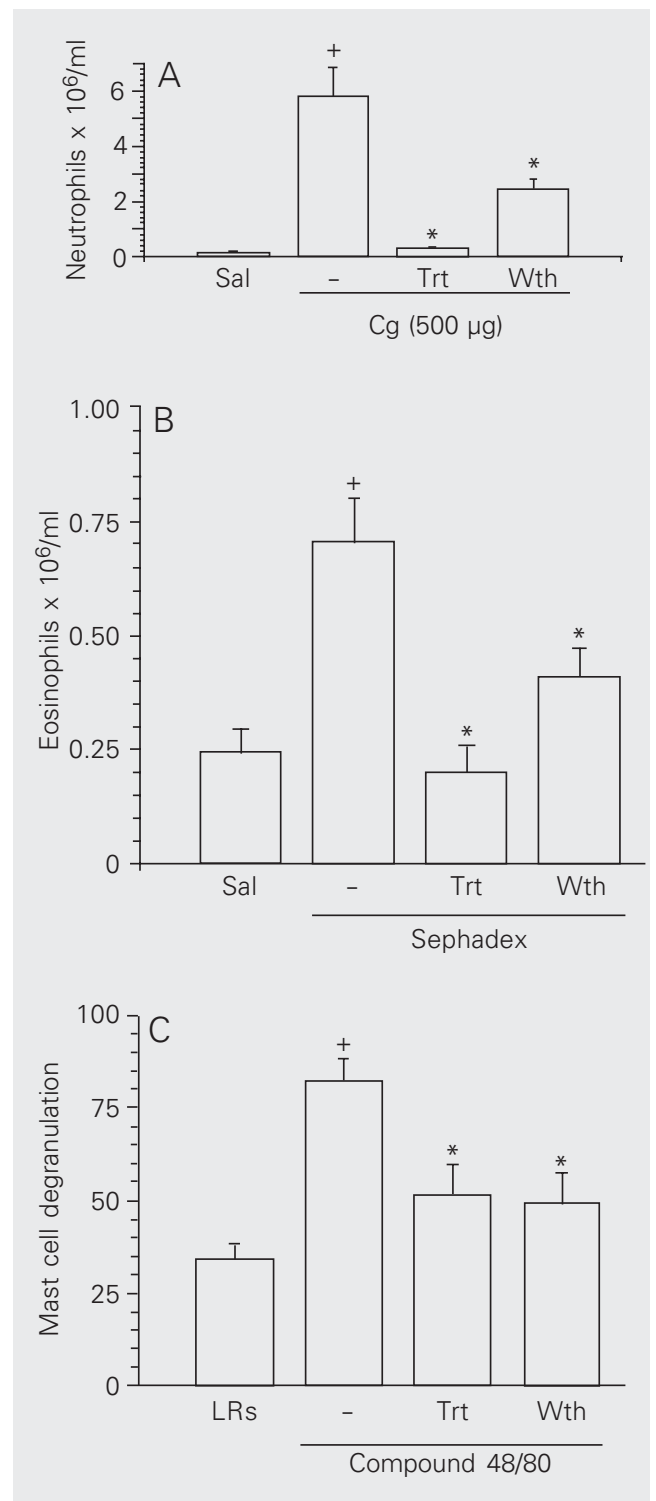


Figure 4. Effect of ethanol inhalation on total leukocyte (A) and neutrophil (B) counts in peripheral blood of normal animals. The leukogram was determined before (day 0) and on the 5th, 9th, 10th, and 10th day $+7 \mathrm{~h}$ of ethanol withdrawal. Each point represents the mean \pm SEM of the number of leukocytes or neutrophils $\times 10^{6} / \mathrm{ml}$ for $5-6$ rats per group. The dashed line is the horizontal extension of the values obtained on day $0 .{ }^{*} \mathrm{P}<0.05$ compared to the control period (day 0; ANOVA for repeated measurements, followed by the Bonferroni test). strated that acute alcohol intoxication inhibits neutrophil chemotaxis and suppresses the responses to various inflammatory stimuli (23). However, there are few studies correlating the effects of ethanol on the inflammatory process using model of physical dependence on ethanol induced by inhalation and the withdrawal syndrome model. In the present study, we also used the inhalation route because this route is considered to be an efficient method to obtain a constant serum ethanol concentration (14). A sign of withdrawal (convulsions on handling) here detected appeared to reach a peak at $7 \mathrm{~h}$, but high values were also obtained between 6 and $11 \mathrm{~h}$ (data not shown), a result closely similar to the findings of previous reports $(14,18)$. Although these results indicated that the dose was sufficiently high to produce dependence on ethanol, they do not allow the conclusion that there is a relationship between abstinence duration and changes in
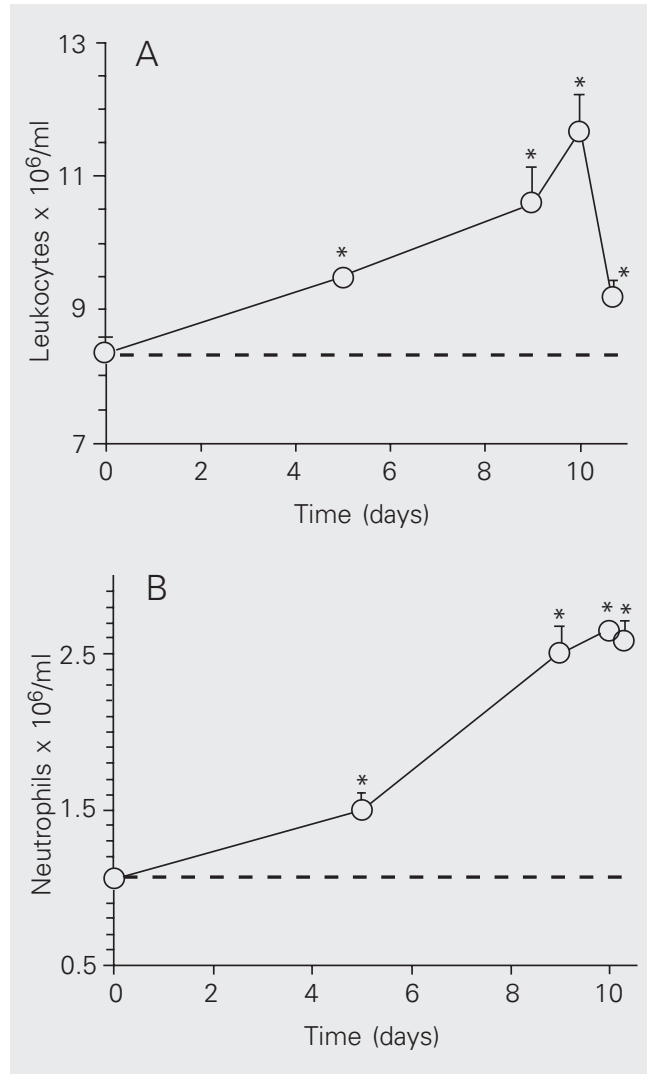

the magnitude of the inflammatory response.

In the present study we present evidence showing that long-term ethanol administration has an anti-inflammatory effect. Carrageenan- and dextran-induced paw edema was significantly reduced by long-term ethanol inhalation. Both kinds of edema were also reduced in the withdrawal group. Carrageenan and dextran have been shown to increase vascular permeability through different mechanisms. While dextran induces fluid accumulation due to mast cell degranulation with little protein and few neutrophils, carrageenan induces a protein-rich exudate containing a large number of neutrophils (22). Since ethanol inhalation showed an inhibitory effect on carrageenan and dextran-induced edema, we assume that ethanol treatment reduces both polymorphonuclear leukocytedependent and mast cell-dependent edema.

We also showed that long-term ethanol administration in both treated and withdrawal groups significantly inhibited mast cell degranulation induced by compound $48 / 80$. Our data are consistent with those that showed that in vitro ethanol treatment attenuated IgE-induced degranulation of mouse bone marrow-derived mast cells (13). Thus, the impairment of mast cell degranulation seen in ethanol-treated animals could account for the reduction of paw edema induced by dextran, a well-known edema dependent on histamine and other mediators released from mast cells. Furthermore, ethanol markedly reduced the increased vascular permeability caused by histamine and serotonin in Wistar rats (24), an effect that could further explain the decrease in rat paw edema in ethanoltreated animals.

The present results also show that ethanol treatment inhibited the carrageenan-induced neutrophil migration and the Sephadex-induced eosinophil migration to a 6day-old air pouch and peritoneal cavity, respectively, in both the treated and withdrawn groups. The inhibition of neutrophil migration in response to ethanol treatment is con- 
sistent with the reduction of carrageenan edema, since the vascular permeability which characterizes this edema is a known polymorphonuclear-dependent inflammatory event. It has been demonstrated that carrageenan-induced neutrophil migration occurs by a process dependent on the release of chemotactic mediators by resident cells $(25,26)$. Thus, it is possible that the inhibitory effect of ethanol on carrageenan-induced neutrophil recruitment could occur by an inhibitory action on the release of chemotactic agents such as leukotriene $B_{4}$, TNF- $\alpha$, interleukin 1 or 8 from macrophages and/or mast cells. In agreement with this possibility is the fact that it has been demonstrated that ethanol pretreatment inhibits both interleukin 8 and TNF- $\alpha$ production by human mononuclear cells in vitro (27). Additionally, it has been shown that the production of inflammatory cytokines by alveolar macrophages from long-term ethanol-fed mice is reduced, potentially contributing to lung infections $(9,10)$. Similarly, the Sephadex-induced eosinophil recruitment was also inhibited in both the treated and withdrawn groups. As is the case for neutrophil migration, Sephadex-induced eosinophil migration occurs by a process dependent on the release of chemotactic substances by resident cells $(28,29)$, which may be inhibited by long-term ethanol administration.

In order to investigate the relationship between polymorphonuclear production and its delivery to the site of inflammation, we performed a leukogram over the 10-day period of ethanol inhalation and $7 \mathrm{~h}$ after ethanol withdrawal. A progressive increase in the number of neutrophils in peripheral blood was detected, with a peak on the tenth day. Seven hours after the interruption of ethanol exposure, the leukocyte count was significantly decreased, probably due to a decrease in mononuclear cell count (data not shown). This result suggests that the inhibition of neutrophil migration is not attributable to the induction of peripheral neutropenia. There- fore, a possible explanation for the increase in circulating polymorphonuclear neutrophils in intoxicated animals could be a decreased adherence, leading to demargination or changes in vascular permeability which could prevent cell movement out of the vascular compartment. Thus, we can suggest at least two ways by which ethanol might inhibit neutrophil recruitment: 1) interference with chemotactic mediator release, and 2) downregulation of adhesion molecule expression in polymorphonuclear cells or endothelial cells. Consistent with the latter hypothesis, it has been shown that the FMLP-induced upregulation of Mac-1, an adhesive glycoprotein involved in neutrophil migration, is inhibited by ethanol (30). The two mechanisms suggested here could also be responsible for ethanol-mediated inhibition of eosinophil migration and of other cells involved in the immune-inflammatory response.

Some evidence suggests that the antiinflammatory effect of ethanol is not significant under in vivo conditions of long-term alcohol consumption (31). The authors of the latter study suggested that neutrophils have the ability to overcome ethanol inhibition under conditions of prolonged ethanol treatment. However, our data demonstrate that the effects of long-term ethanol administration on neutrophils and other blood elements persist even $7 \mathrm{~h}$ after interruption of ethanol exposure. These differing results may be explained by the fact that the inhalation method used here permits a continuous intoxication and a more stable blood alcohol concentration than the model of long-term oral consumption. Since during the withdrawal period, the inhibition of the inflammatory response seems to be recovering, especially regarding the polymorphonucleardependent events, it is possible that these effects observed during withdrawal are lasting consequences of ethanol treatment.

Thus, both the cellular and vascular components of the inflammatory response are compromised by long-term exposure to etha- 
nol, and this impairment persists during the withdrawal period. Finally, our data on paw edema, leukocyte migration and mast cell degranulation from withdrawal group suggest that the immune system may still be depressed throughout the period following alcohol consumption.

\section{Acknowledgments}

\author{
We thank Dr. Albert Leyva from Instituto \\ de Tecnologia Química, CSIC-UPV, Valen- \\ cia, Spain, for reading the manuscript.
}

\section{References}

1. Gluckman SJ \& MacGregor RR (1978). Effect of acute alcohol intoxication on granulocyte mobilization and kinetics. Blood, 52: 551-559.

2. Gluckman SJ, Dvorak VC \& MacGregor RR (1977). Host defenses during prolonged alcohol consumption in a controlled environment. Archives of Internal Medicine, 137: 1539-1543.

3. Brayton RG, Stokes PE, Schwartz MS \& Louria DB (1970). Effect of alcohol and various diseases on leukocyte mobilization, phagocytosis and intracellular bacterial killing. New England Journal of Medicine, 282: 123-128.

4. Hunt JD, Robert EG, Zieske AW, Bautista AP, Bukara M, Lei D, Shellito JE, Nelson S, Kolls JK \& Skrepnik N (2000). Orthotopic human lung carcinoma xenografts in BALB/C mice immunosuppressed with anti-CD4 monoclonal antibodies and chronic alcohol consumption. Cancer, 88: 468-479.

5. Szabo G (1999). Consequences of alcohol consumption on host defence. Alcohol and Alcoholism, 34: 830-841.

6. Patel M, Keshavarzian A, Kottapalli V, Badie B, Winship D \& Fields JZ (1996). Human neutrophil functions are inhibited in vitro by clinically relevant ethanol concentrations. Alcoholism, Clinical and Experimental Research, 20: 275-283.

7. MacGregor RR (1990). In vivo neutrophil delivery in men with alcoholic cirrhosis is normal despite depressed in vitro chemotaxis. Alcoholism, Clinical and Experimental Research, 14: 195-199.

8. Castro A, Lefkowitz DL \& Lefkowitz SS (1993). The effects of alcohol on murine macrophage function. Life Sciences, 52: 15851593.

9. Standiford TJ \& Danforth JM (1997). Ethanol feeding inhibits proinflammatory cytokine expression from murine alveolar macrophages ex vivo. Alcoholism, Clinical and Experimental Research, 21: 12121217.

10. Nelson S, Bagby G \& Summer WR (1989). Alcohol suppresses lipopolysaccharide-induced tumor necrosis factor activity in serum and lung. Life Sciences, 44: 673-676.

11. Spolarics Z, Spitzer JJ, Wang JF, Xie J, Kolls J \& Greenberg S (1993). Alcohol administration attenuates LPS-induced expression of inducible nitric oxide synthase in Kupffer and hepatic endothelial cells. Biochemical and Biophysical Research Communications, 197: 606-611.

12. Syapin PJ (1995). Ethanol inhibition of inducible nitric oxide synthase activity in C6 glioma cells. Alcoholism, Clinical and Experimental Research, 19: 262-267.

13. Toivari M, Maki T, Suutarla S \& Eklund KK (2000). Ethanol inhibits IgE-induced degranulation and cytokine production in cultured mouse and human mast cells. Life Sciences, 67: 2795-2806.

14. Ferko AP \& Bobyock E (1977). Induction of physical dependence in rats by ethanol inhalation without the use of pyrazole. Toxicology and Applied Pharmacology, 40: 269-276.
15. Dahchour A \& De Witte P (2003). Excitatory and inhibitory amino acid changes during repeated episodes of ethanol withdrawal: an in vivo microdialysis study. European Journal of Pharmacology, 459: 171-178.

16. Granato A, Di Rocco F, Zumboa A, Toesca A \& Giannetti S (2003). Organization of cortico-cortical associative projections in rats exposed to ethanol during early postnatal life. Brain Research Bulletin, 60: 339-344.

17. Buck KJ, Rademacher BLS, Metten P \& Crabbe JC (2002). Mapping murine loci for physical dependence on ethanol. Psychopharmacology, 160: 398-407.

18. Goldstein DB (1972). Relationship of alcohol dose to intensity of withdrawal signs in mice. Journal of Pharmacology and Experimental Therapeutics, 180: 203-215.

19. Edwards JC, Sedgwick AD \& Willoughby DA (1981). The formation of a structure with the features of synovial lining by subcutaneous injection of air: an in vivo tissue culture system. Journal of Pathology, 134: 147-156

20. Ribeiro RA, Flores CA, Cunha FQ \& Ferreira SH (1991). IL-8 causes in vivo neutrophil migration by a cell-dependent mechanism. Immunology, 73: 472-477.

21. Landucci EC, Antunes E, Donato JL, Faro R, Hyslop S, Marangoni S, Oliveira B, Cirino G \& De Nucci G (1995). Inhibition of carrageenininduced rat paw oedema by crotapotin, a polypeptide complexed with phospholipase A2. British Journal of Pharmacology, 114: 578583

22. Lo TN, Almeida AP \& Beaven MA (1982). Dextran and carrageenan evoke different inflammatory responses in rat with respect to composition of infiltrates and effect of indomethacin. Journal of Pharmacology and Experimental Therapeutics, 221: 261-267.

23. Levallois C, Rouahi N, Balmes JL \& Mani JC (1989). Effects of ethanol in vitro on some parameters of the immune response. Drug and Alcohol Dependence, 24: 239-244.

24. Oga S, Hanada S, Basile AC, Sertie JA \& Yasaka WJ (1983). Effects of ethanol on experimental inflammation. Polish Journal of Pharmacology and Pharmacy, 35: 201-207.

25. De Souza GE \& Ferreira SH (1985). Blockade by antimacrophage serum of the migration of PMN neutrophils into the inflamed peritoneal cavity. Agents and Actions, 17: 97-103.

26. Kowanko IC, Gordon TP, Rozenbilds MA, Brooks PM \& RobertsThomson PJ (1986). The subcutaneous air pouch model of synovium and the inflammatory response to heat aggregated gammaglobulin. Agents and Actions, 18: 421-428.

27. Arbabi S, Garcia I, Bauer GJ \& Maier RV (1999). Alcohol (ethanol) inhibits IL-8 and TNF: role of the p38 pathway. Journal of Immunology, 162: 7441-7445.

28. Lopez AF, Williamson DJ, Gamble JR, Begley CR, Harlan JM, Klebanoff SJ, Waltersdorph A, Wong G, Clark SC \& Vadas MA 
(1986). Recombinant human granulocyte-macrophage colony-stimulating factor stimulates in vitro mature human neutrophil and eosinophil function, surface receptor expression, and survival. Journal of Clinical Investigation, 78: 1220-1228.

29. Walsh GM, Hartnell A, Wardlaw AJ, Kurihara K, Sanderson CJ \& Kay $A B$ (1990). IL-5 enhances the in vitro adhesion of human eosinophils, but not neutrophils, in a leukocyte integrin (CD11/18)-depend- ent manner. Immunology, 71: 258-265.

30. MacGregor RR, Safford M \& Shalit M (1988). Effect of ethanol on functions required for the delivery of neutrophils to sites of inflammation. Journal of Infectious Diseases, 157: 682-689.

31. Corberand JX, Laharrague PF \& Fillola G (1989). Human neutrophils are not severely injured in conditions mimicking social drinking. Alcoholism, Clinical and Experimental Research, 13: 542-546. 